\title{
reviscafuences
}

ISSN: 1575-7072 | e-ISSN: 2172-7775

Páginas: $65-74$

Recibido: 2019-03-13

Revisado: 2019-09-16

Aceptado: 2019-11-27

Preprint: 2020-04-20

Publicación Final: 2020-06-15 www.revistascientificas.us.es/index.php/fuentes/index

DOI: https://doi.org/10.12795/revistafuentes.2020.v22.i1.06

\section{Estudio etiológico de los errores del alumnado francófono de catalán nivel B1}

\author{
Etiological Study of the Errors Made by Francophone Learners of Catalan at B1 \\ Leve
}

\begin{abstract}
Este artículo presenta los resultados de un estudio etiológico de diferentes tipos de errores en la producción escrita de alumnado francófono de catalán de nivel B1 (Umbral). El objetivo de esta investigación es detectar recurrencias en los tipos de errores y así contribuir a la reflexión sobre las estrategias didácticas para la enseñanza de aspectos concretos del catalán B1 a estudiantes cuya L1 es el francés. Para ello, se analizó un corpus de redacciones siguiendo el modelo de examen oficial de lengua catalana del Institut Ramon Llull. La metodología utilizada permitió la detección y posterior análisis de cada error a partir de criterios descriptivos y etiológicos. Este artículo presenta resultados cuantitativos y cualitativos desde un punto de vista principalmente etiológico (causas interlingüísticas e intralingüísticas), lo que permite la observación de ciertas tendencias y la formulación de generalizaciones. Finalmente, se proponen posibles vías de trabajo pedagógico concreto para la enseñanza de estos aspectos específicos del catalán.
\end{abstract}

Resumen

\section{Abstract}

This article presents the results of an etiological study of different types of errors in the written production of francophone learners of Catalan at B1 (Threshold) level. The main aim of this research is to identify error patterns in order to reflect on and develop pedagogical strategies to support learners of Catalan whose L1 is French. In order to do so, a corpus of written productions based on the official exams of Institut Ramon Llull was analysed. Errors were analysed and classified using descriptive and etiological criteria. This article presents both quantitative and qualitative results from an etiological perspective (interlinguistic and intralinguistic causes). This has allowed for the identification of tendencies and the formulation of generalisations. Finally, a series of pedagogical recommendations for the teaching of these is presented.

\section{Palabras clave / Keywords}

Catalán, Enseñanza de una segunda lengua, Redacción, Análisis cuantitativo, Análisis cualitativo, Ciencias de la educación, Examen, Estudiante adulto.

Catalan, Second language instruction, Writing, Quantitative analysis, Qualitative analysis, Educational sciences, Examinations, Adult students. 


\section{Introducción}

Los países y las comunidades animan cada vez más a la ciudadanía a aprender lenguas extranjeras, puesto que ello se considera un elemento clave para su desarrollo y su plenitud (Eurostat, 2016). El Análisis de errores (AE) puede contribuir de manera notable a la mejor comprensión de los errores del alumnado y, por tanto, a la reflexión y puesta en práctica de estrategias didácticas para corregir errores recurrentes, lo que redundará en un desarrollo más óptimo de la competencia comunicativa del alumnado de lenguas extranjeras. Partiendo de la idea de interlengua (IL) como resultado de una competencia transitoria del alumnado de lenguas (Fernández Jódar, 2006, p. 10), el proyecto "Competència Comunicativa Transitòria i Aprenentatge de Llengües", de la Université TÉLUQ (Quebec), se centra en el análisis y clasificación etiológica de los errores detectados en redacciones de alumnado francófono de catalán de nivel B1, con el fin de explicitar comentarios u observaciones pedagógicas, y evitar o corregir de este modo los errores recurrentes. Así pues, el objetivo final del proyecto es contribuir a la evolución de la IL hacia un dominio más asentado del catalán como lengua extranjera por parte de este tipo de alumnado. Los errores analizados aquí son considerados, pues, como una oportunidad para la mejora de la competencia para la comunicación en lengua extranjera (Astolfi, 1997, 2015).

\section{Fundamentación teórica}

En el contexto específico en el que se ha desarrollado esta investigación (alumnado universitario procedente del microprograma de primer ciclo de Lengua y Cultura Catalanas de la Université de Montréal que ha superado el examen oficial del B1 de catalán del Institut Ramon Llull), la enseñanza del catalán como lengua extranjera se realiza actualmente desde una perspectiva comunicativa basada en tareas y funciones comunicativas (Ellis, 2003): se plantea al alumnado diferentes situaciones comunicativas y se trabaja cómo asegurar una comunicación eficaz según los objetivos establecidos para cada tarea. Algunos manuales que permiten esta perspectiva son Veus (Mas, Vilagrasa Grandia y Bastons i Vilallonga, 2009) y A punt (Verdés Prieto, Álvarez Verger, Birello, Mas Prats y Pérez Ventayol, 2018). Dichos manuales se estructuran en capítulos sobre cómo presentarse, cómo hablar de los otros, cómo negociar lo que nos gusta y lo que no nos gusta, cómo obtener indicaciones para llegar a un destino, etc. En el caso de nuestro corpus, obtenido a partir de producciones escritas siguiendo el modelo de examen oficial del Institut Ramon Llull para el nivel B1 del Marco común europeo de referencia para las lenguas (MCER) (Consejo de Europa, 2001, 2018), las situaciones comunicativas propuestas y, por tanto, las producciones escritas del alumnado, se corresponden con esta visión metodológica.

Partiendo de la conceptualización de competencia comunicativa del MCER (Consejo de Europa, 2001) como una macrocompetencia compuesta a su vez por tres subcompetencias (a saber, lingüística, sociolingüística y pragmática), el análisis realizado para esta investigación se centra en uno de los tres componentes del modelo CAF (complexity, accuracy, fluency). El modelo CAF viene referido a los criterios de complejidad, precisión y fluidez en el uso de la lengua (Ellis, 2003, 2008; Ellis y Barkhuizen, 2005; Housen, Kuiken y Vedder, 2012; Skehan, 1998). Esta investigación se centra en la precisión que, según el MCER (Consejo de Europa, 2018, p. 150), concierne la capacidad de determinar cómo formular lo que se quiere decir. El presente estudio se centra en este criterio de precisión, tanto en el nivel puramente lingüístico (niveles ortográfico, morfosintántico y léxico-semántico) como en el pragmático-discursivo.

En lo que respecta al $\mathrm{AE}$, se trata de una disciplina de investigación que agrupa diversas perspectivas e investigaciones. Erdogan (2005, p. 269) propone tres características comunes que permiten describir de forma general las investigaciones vinculadas al $\mathrm{AE}$ en el aprendizaje de lenguas extranjeras. Según Erdogan, el $\mathrm{AE}$ identifica las estrategias utilizadas para los estudiantes de una lengua; pretende explicar por qué los estudiantes se equivocan; y determina las dificultades comunes en el aprendizaje contribuyendo al desarrollo de los recursos y materiales para solventarlas (Erdogan, 2005, p. 269).

Como se ha avanzado anteriormente, la investigación en $\mathrm{AE}$ que se ha realizado para el presente estudio parte, además, de la idea de la interlengua (IL), tal y como la propone Fernández Jódar (2006):

La IL [...] es un sistema lingüístico mental recreado por cada aprendiz para expresar en una L2 aquello que podría expresar en su L1. Con tal fin recurre a veces a la L1 (transferencias) o realiza hipótesis que pueden ser correctas o incorrectas, sobre la L2. Este sistema lingüístico mental no debe ser considerado como erróneo sino como un sistema evolutivo entre la L1 y la L2, con 
características de ambas y propias al mismo tiempo, y que refleja la competencia comunicativa transitoria del aprendiz. (p. 10)

Desde esta perspectiva se entiende que el conocimiento del error proporciona, pues, una oportunidad para mejorar los procesos de enseñanza-aprendizaje de las lenguas extranjeras.

Uno de los aspectos más útiles del AE para el campo de la investigación y su aplicación a la práctica educativa es el conjunto de reflexiones en torno a los errores, así como el abanico de propuestas de tipologías o taxonomías de errores, ya que se trata de una etapa indispensable que precede al tratamiento de los datos del corpus y a la obtención de los resultados. Podemos encontrar, pues, propuestas de tipologías muy diversas, centradas en: un tipo de error concreto o errores de forma global; diferentes criterios según los objetivos de la investigación; la lengua estudiada en cuestión o independientemente de esta.

Puesto que el foco de este estudio se encuentra en la descripción lingüística de los errores y en su etiología (causas subyacentes que han provocado el error), se ha diseñado una taxonomía que permite observar las características de los errores en las redacciones del alumnado desde el punto de vista de la lengua como sistema y las posibles explicaciones sobre su aparición. Esta taxonomía, diseñada ad hoc para el presente análisis, se ha construido a partir de distintas propuestas teóricas que se presentan a continuación. La Tabla 1 en los Anexos muestra los campos directamente relacionados con el análisis descriptivo de los errores, mientras que la Tabla 2 de los Anexos presenta la clasificación de los aspectos relacionados con el análisis etiológico de los errores.

En cuanto a la taxonomía de descripción lingüística, la propuesta de campos utilizada en la presente investigación para la anotación del nivel lingüístico es lo más detallada posible (Tabla 1): se separan las opciones de ortografía en dos subtipos (con y sin consecuencias fonéticas), y se distingue también entre morfología y sintaxis. Puesto que se trata de una primera aproximación al corpus, se prefiere realizar una anotación detallada y considerar posteriormente si se pueden llevar a cabo generalizaciones y/o reducciones de etiquetas en investigaciones posteriores. En este sentido, se ha optado por las propuestas de Corder (1973) y Ellis (1997). Se añade solamente la distinción entre "selección de un elemento erróneo" y "forma errónea", con el objetivo de observar si la especificación es pertinente.

En lo que respecta a la taxonomía referente a la etiología (Tabla 2), los autores consultados coinciden en la distinción entre causas interlingüísticas e intralingüísticas. Dentro de las causas interlingüísticas, se ha considerado apropiado detallar el origen. En cuanto a las causas intralingüísticas, las propuestas estudiadas presentan clasificaciones similares y variadas a la vez. En la clasificación utilizada para el presente estudio, se ha optado por escoger las etiquetas que son lo suficientemente generales y, a su vez, claras para la anotación. No se han tenido en cuenta otras etiquetas de clasificación (evolutivas, por ejemplo) por no ser apropiadas para el corpus en cuestión.

\section{Método}

Esta investigación tiene una vertiente descriptiva (se detectan y describen lingüísticamente los errores), otra analítica (se presenta el análisis etiológico de dichos errores) y otra aplicada (se proponen una serie de propuestas didácticas para reducir los errores).

El corpus analizado se compone de un total de 17 redacciones realizadas para el examen oficial de B1 de catalán del Institut Ramon Llull. Según el MCER (Consejo de Europa, 2001, 2018), el nivel B1 se corresponde con un inicio de la autonomía del alumnado en la lengua aprendida; en el caso de la producción escrita, indica que el alumnado sabe expresar lo esencial de lo que desea comunicar. La actividad que el alumnado debía realizar consistía en escribir una carta informal a una persona cercana explicando sus primeras experiencias durante una estancia en el extranjero (alojamiento, cursos o prácticas, características del lugar, personas que había conocido, etc.) e invitando a esa persona a hacerle una visita. En el MCER (Consejo de Europa, 2018), esta actividad viene clasificada como una actividad de escritura creativa dentro de la producción escrita general (Consejo de Europa, 2018, p. 71). La escritura creativa incluye la expresión personal e imaginaria en un abanico de tipos de textos. Los descriptores del nivel B1 para la escritura creativa en el MCER son los siguientes:

1. Puede escribir descripciones detalladas no complejas sobre una gama amplia de temas familiares en el marco de su ámbito de interés.

2. Puede explicar experiencias describiendo sus sentimientos y sus reacciones en un texto simple y articulado. 
3. Puede escribir la descripción de un acontecimiento, de un viaje reciente, real o imaginado.

4. Puede contar una historia.

A su vez, la actividad planteada también muestra al final una vertiente de interacción simulada, puesto que se trata de una carta en la que se ha de invitar al destinatario a visitar al remitente. Por lo tanto, desde el punto de vista de la interacción, la actividad también se inscribe en la interacción escrita general (correspondencia) (Consejo de Europa, 2018, p. 85), en la que se evalúa si el alumnado puede escribir cartas personales describiendo con detalle experiencias, sentimientos y acontecimientos, además de obtener respuesta a preguntas realizadas (Consejo de Europa, 2018, p. 97).

El corpus se ha seleccionado teniendo en cuenta las producciones del alumnado cuya L1 es el francés. Se trata de alumnado universitario procedente del microprograma de primer ciclo de Lengua y Cultura Catalanas de la Université de Montréal que ha superado el examen oficial de B1 de catalán del Institut Ramon Llull. De este alumnado, el $11,76 \%$ solo habla la $L 1$, mientras que la mayoría $(70,59 \%)$ habla dos o más lenguas. Si se consideran de forma independiente las diferentes lenguas aprendidas (excluyendo el catalán), el inglés y el español son las más habladas por el alumnado (Tabla 3 en los Anexos).

Por otro lado, la mayor parte del alumnado conoce el inglés y/o el español (Tabla 4 en los Anexos). En cuanto al inglés, en este contexto su aprendizaje comienza al iniciar los estudios de primaria, a la edad de 6 años. Por lo que respecta al español, en algunos casos, el alumnado ha desarrollado algo de competencia en lengua española en asignaturas optativas durante la Educación Secundaria. Además, el alumnado en cuestión está cursando Études hispaniques (asimilable a Filología Hispánica) y ha completado el microprograma de primer ciclo de Lengua y Cultura Catalanas que le da acceso al B1 de catalán.

Con respecto a las técnicas e instrumentos utilizados para esta investigación, se ha realizado una anotación manual de los errores, seguida de un análisis estadístico mediante el programa SPSS. Concretamente, el número de errores efectuados para cada uno de los subtipos está resumido mediante frecuencias y porcentajes, dentro del conjunto de 237 errores detectados en el corpus. Asimismo, se han obtenido tablas de frecuencias cruzadas para estudiar la relación entre los dos tipos de errores (interlingüísticos e intralingüísticos), y correlaciones de Spearman para medir la asociación entre el número de errores de cada tipo por estudiante. Dichas correlaciones van del -1 al 1 (siendo 0 la no correlación) y se consideran estadísticamente significativas cuando la significación es menor de 0,05. Cabe mencionar que ninguno de los análisis estadísticos realizados tiene como hipótesis la normalidad, pero que no se han detectado datos extremos.

El procedimiento global de análisis utilizado ha sido el siguiente:

1. Detección de los errores en cada una de las redacciones que conforman el corpus.

2. Anotación descriptiva de cada error:

Nivel lingüístico.

Fenómeno.

3. Anotación etiológica de cada error:

Posibles causas interlingüísticas.

Posibles causas intralingüísticas.

4. Análisis cuantitativo de los resultados (estadística).

5. Análisis cualitativo de los resultados.

Finalmente, a partir de las tendencias de errores observadas en los resultados, se han propuesto una serie de estrategias didácticas para mejorar los puntos débiles del alumnado francófono de catalán.

\section{Resultados y discusión}

En esta sección se presentan los resultados, tanto cuantitativos como cualitativos, y la discusión de estos.

\subsection{Número y tipología de errores}

En el análisis se ha detectado un total de 237 errores, que han sido clasificados según su causa (Tabla 5, Anexos). Las causas intralingüísticas son las predominantes (168 casos en total) en los errores cometidos en este nivel de lengua, tanto si son causa única del error (83) como si se combinan con las interlingüísticas (85), frente a las causas exclusivamente interlingüísticas (50). Esto significa que, en este nivel, los errores más 
comunes son los que provienen de interferencias con lo aprendido para la misma lengua (el catalán), frente a los errores que proceden exclusivamente de interferencias con lo aprendido para otras lenguas (bien la L1 u otras lenguas aprendidas). A continuación se presentan ejemplos del corpus que muestran errores por causas exclusivamente intralingüísticas ( 1 y 2 ) y errores por causas solamente interlingüísticas (3 y 4):

(1) Li agrada també la história [història] i sovint anem al museu junts.

(2) Visc a [en] un petit pis a l'ultima planta d'un edifici del barri central, molt a prop de l'escola.

(3) És una gran casa que té dues plantes, més aviat quadrada. Com que és espaiosa, hi tinc la meva propera [pròpia] habitació al segon pis.

(4) Els dies de la setmana [Entre setmana] és tranquil, en canvi els caps de setmana la gent fa molt soroll.

En el ejemplo (1) el tipo de tilde utilizada no es el correcto, aunque habitualmente las tildes en palabras esdrújulas son graves en catalán. En el ejemplo (2), a diferencia de lo que es habitual como preposición para el verbo "vivir" en catalán, el uso de la preposición "a" en lugar de "en" no es correcto por aparecer delante del determinante indefinido "un". Ambos errores proceden de interferencias de lo ya aprendido para el catalán (que puede haber sido parcialmente aprendido o aplicado de manera incorrecta, como veremos). Por otro lado, en el ejemplo (3) se encuentra el uso de "propera" en lugar de "pròpia" para referirse a lo que en francés sería "propre" y, en el ejemplo (4), "els dies de la setmana" en lugar de "entre setmana" para "les jours de la semaine". Como puede verse, se trata de una influencia del francés, que tiene lugar principalmente en el nivel léxico-semántico produciendo calcos.

Sin embargo, como ya se ha apuntado, el mayor número de errores encontrados en el corpus combinan causas intralingüísticas e interlingüísticas (85 en total). Este es el caso del ejemplo (5):

(5) Desitjo que tinguis molt bon temps i aprofitis de [de] les teves vacances.

El uso de la preposición "de" con "aprofitar" existe en catalán, pero solamente con el sentido de "sacar provecho de algo con astucia o abuso", y no de "sacar el máximo provecho o rendimiento a algo". Para expresar esto último, se omitiría la preposición y el verbo sería transitivo ("desitjo que aprofitis les teves vacances"). Se trata de un error de tipo intralingüístico (hipergeneralización por falsa analogía) que, además, podría estar apoyado también por interferencia de la L1 ("je souhaite que tu profites de tes vacances"). Así pues, se observa que en el nivel B1 es común que los dos tipos de estrategias, intralingüísticas e interlingüísticas, se apoyen mutuamente cuando el alumnado reflexiona sobre cómo escribir algo, incidiendo así en su toma de decisiones: se encuentra con dos ejemplos o argumentos procedentes de dos o más lenguas que apoyan una determinada formulación. Sin embargo, estos modelos no garantizan que la formulación sea correcta para la lengua en cuestión. Para conocer más sobre el peso que tienen unas lenguas sobre otras cuando operan los dos tipos de estrategias, es necesario realizar estudios más amplios sobre este aspecto concreto.

Por lo tanto, aunque la L1 u otras lenguas continúan teniendo influencia en las producciones escritas del alumnado a este nivel, los datos muestran que las estrategias exclusivamente intralingüísticas son las más habituales en este estadio, aunque algunas de ellas no se utilizan correctamente, provocando errores. De hecho, Fernández (1997, p. 48) ya trata sobre el diferente uso de estrategias interlingüísticas e intralingüísticas dependiendo del estadio de aprendizaje de una lengua. También, en el ámbito de los errores morfológicos, Alexopoulou (2006, p. 33) establece una relación inversa entre los errores interlingüísticos y los intralingüísticos a medida que las competencias lingüísticas mejoran (los primeros serían más frecuentes al principio).

\subsection{Etiología de los errores: causas exclusivamente interlingüísticas}

En una clasificación más detallada de los errores por causas exclusivamente interlingüísticas (Tabla 6, Anexos), puede observarse que la interferencia de la $L 1$ (el francés en nuestro corpus) predomina claramente $(58 \%)$ y de manera significativa $(p=0,009)$ frente a las interferencias de otra lengua aprendida $(30 \%)$ y a las interferencias que pueden ser causadas por ambas (L1 u otra lengua aprendida) (12\%). A continuación, se muestran ejemplos de errores por interferencia de la $L 1$ (ejemplos 6,7 y 8), por interferencia de otra lengua aprendida $(9,10,11$ y 12$)$ y por interferencia de la L1 u otra lengua aprendida (13):

(6) També m'he [m'] fet una amiga al curs. 
(7) A més, la cuina no és gaire bona i els anglès [anglesos] són més aviat avorrits.

(8) Voldria que coneguis la meva família d'acoll [d'acollida] perquè és fantàstica!

(9) És força divertit, sempre explica acudits amb l'accent del nord del país, on vivía [vivia] abans.

(10) Tinc clases [classes] al matí i tallers de laboratori a la tarda.

(11) El comparto [comparteixo] amb un estudiant mexicà molt trempat.

(12) I saps, segons sembla, estem buscant més empleats a la feina i potser et podria presentar al [a el] meu cap!

(13) El climat [clima] és agradable.

El ejemplo (6) es a nivel morfológico, con la equivalencia en francés de "je me suis fait une amie", que utiliza la versión pronominal del verbo. El ejemplo (7) presenta problemas a nivel sintáctico por la falta de concordancia de número; aunque dicha concordancia existe en francés, la forma no cambia para la palabra en cuestión en ese idioma ("I'Anglais/les Anglais") y no parece pues que el estudiante detecte fácilmente el problema aun siendo capaz de concordar el resto de las palabras de la oración. El ejemplo (8) presenta un error a nivel léxico-semántico: la influencia de la expresión en francés "famille d'accueil" es evidente con el uso de una palabra que no existe en catalán ("acoll"). La influencia de la L1 parece producirse, pues, en distintos niveles del sistema lingüístico.

Los casos (9) y (10) muestran errores ortográficos con consecuencias fonéticas: el primero debido a un uso incorrecto de la tilde y el segundo a causa de la omisión de una "s". Por lo que respecta al ejemplo (11), la conjugación del verbo "compartir" es incorrecta y, en cuanto al (12), el uso de la preposición "a" para introducir el complemento directo es también incorrecto. Todos estos ejemplos tienen en común el hecho que no habrían sido errores de tratarse de oraciones en lengua española. Por tanto, podemos considerar que este tipo de errores se producen por influencia del español y no, por ejemplo, del inglés, ya sea por razones de orden de aprendizaje o nivel de competencia, o por razones de similitudes estructurales más próximas entre el español y el catalán. Se pueden proporcionar dos posibles explicaciones para este hecho. Por un lado, es posible que, como se ha indicado anteriormente, el español sea una lengua aprendida más recientemente que el inglés y, por tanto, cuyas estructuras y reglas están menos asentadas y delimitadas. Por otro lado, el español es, además, una lengua más próxima y, por tanto, más parecida al catalán, ya que ambas son lenguas romances; en este caso, las diferencias menos evidentes o los matices más similares podrían provocar más confusión. Algunos autores ya indican situaciones similares en comparaciones lingüísticas en otros contextos como, por ejemplo, en la fluidez en expresión oral (Huensch y Tracy-Ventura, 2017).

Finalmente, el ejemplo (13) presenta un calco léxico que podría proceder del inglés ("climate") o del francés ("climat"). Los otros errores detectados en el corpus no permiten discernir (como en el caso 13) si de verdad existe una influencia del inglés u otra lengua, o simplemente se trata una vez más de interferencias de la L1.

\subsection{Etiología de los errores: causas exclusivamente intralingüísticas}

Los errores por causas exclusivamente intralingüísticas son, como se ha apuntado, los más frecuentes que se han detectado en este nivel. Si consideramos una clasificación más detallada de estos errores (Tabla 7 , Anexos), puede verse que casi la mitad de estos errores es consecuencia de la aplicación incompleta de reglas $(43,38 \%)$, que es causa predominante de los errores intralingüísticos de manera significativa $(p=$ $0,012)$. Con menor frecuencia, se encuentra la hipergeneralización por falsa analogía $(27,72 \%)$ y la no aplicación de las restricciones de las reglas $(21,69 \%)$. mientras que la hipercorrección es anecdótica $(7,21 \%)$, lo que implica que no se trata de una estrategia recurrente en este nivel. A continuación, se presentan algunos ejemplos de errores intralingüísticos por aplicación incompleta de las reglas (ejemplos 14 y 15), por hipergeneralización por falsa analogía (16, 17 y 18), por no aplicación de las restricciones de las reglas (19) y por hipercorrección (20):

(14) Aquest pis és molt maco i esta [està] ben comunicat.

(15) Els professors són molt simpàtics $i$ els cursos bastant interessants, però tinc dificultats [per] llevar-me d'hora cada dia.

(16) El pis, que es [es] queda a la tercera planta, és bastant gran i hi toca el sol.

(17) Sabies que no fa sol gairebé mai aquí? No s'acaba de plujar! [No para de ploure!].

(18) Em va espantar veure com condueixen a mà esquerra [per l'esquerra].

(19) Poderíem [Podríem] visitar el parlament i també el museu d'art contemporani.

(20) Si vinguis [vens] a visitar-me te'l presentaré! 
En el ejemplo (14) falta la tilde de la palabra aguda y, en el (15), una preposición para que la estructura "tenir dificultat(s) per + infinitivo" sea correcta. Estos casos han sido clasificados como aplicación incompleta de las reglas. Los errores por aplicación incompleta de las normas suelen ocurrir a nivel ortográfico con consecuencias fonéticas y, dentro de estos, el más común en el corpus analizado es el uso incorrecto de una tilde, como es el caso del ejemplo (14). Por otro lado, los ejemplos (16), (17) y (18) son casos de hipergeneralización por falsa analogía. En (16), se utiliza "quedar-se" en lugar de "quedar"; en (17), se utiliza una forma errónea del verbo "llover" a partir del uso de una regla morfológica de derivación que sería correcta en otros casos y, además, se utiliza también una forma errónea de perífrasis "no s'acaba de", que existe en catalán pero con otro sentido; en (18), se utiliza la expresión "a mà esquerra", que existe en catalán pero con significado diferente al que se quiere expresar. Cabe mencionar que los casos de hipergeneralización por falsa analogía encontrados en el corpus no se corresponden con un nivel del sistema lingüístico en concreto, pero sí tienden a provocar ambigüedades o distracciones en la comunicación. En el ejemplo (19) existe una no aplicación de las restricciones de las reglas, ya que se utiliza una forma regular incorrecta del verbo "poder" en lugar de la forma irregular correcta. Finalmente, el ejemplo (20) es un caso de hipercorrección donde se utiliza el subjuntivo en lugar del indicativo. Los errores por hipercorrección encontrados en el corpus se dan a nivel sintáctico o discursivo, y suponen el uso excesivo de pronombres débiles o del modo subjuntivo. Sin embargo, como se ha dicho anteriormente, son escasos por lo que se necesitaría analizar un corpus más amplio y a niveles superiores para poder corroborar o no dicha tendencia.

\subsection{Etiología de los errores: causas intralingüísticas en su combinación con causas interlingüísticas}

Finalmente, es posible también observar los resultados sobre los errores por causas intralingüísticas en combinación con causas interlingüísticas (Tabla 8, Anexos). En este caso, las combinaciones más comunes y que, además, muestran correlaciones estadísticamente significativas son la aplicación incompleta de las reglas en conjunción con la interferencia de la $L 1(54,12 \% ; p=0,021)$ y la hipergeneralización por falsa analogía también junto con la interferencia de la $L 1(16,47 \% ; p=0,020)$. Otras correlaciones importantes, si bien no son estadísticamente significativas, son la aplicación incompleta de las reglas con interferencia de otra lengua aprendida $(14,12 \%)$ y con la interferencia de la $L 1 \mathrm{u}$ otra lengua aprendida $(8,23 \%)$. Para todos los casos de errores intralingüísticos, la primera combinación más frecuente es siempre la interferencia de la L1. La influencia de la L1 sigue siendo, pues, una causa importante de los errores en este nivel. Además, como ya han anticipado los resultados anteriores, la estrategia intralingüística más común en este nivel es la aplicación incompleta de las reglas, seguida de la hipergeneralización por falsa analogía y, en mucha menor medida en este caso, de la no aplicación de las restricciones de las reglas. Por último, no existe ningún caso de hipercorrección dentro del conjunto de errores que combinan causas intralingüísticas e interlingüísticas. A continuación, se presentan ejemplos de errores por aplicación incompleta de reglas en combinación con interferencia también de la L1, que se producen mayoritariamente a nivel sintáctico (ejemplos 21 y 22) y a nivel ortográfico con consecuencias fonéticas (23 y 24):

(21) Tot i que els sopars són cars en els restaurant [restaurants], sempre hi vaig perquè l'empresa paga.

(22) A més, la cuina no és gaire bona i els anglès [anglesos] són més aviat avorrits.

(23) T'enyoro i a més estic segur que t'encantara [t'encantarà] la ciutat i la gent d'aquí.

(24) La Lola és simpatica [simpàtica] i ens avenim molt.

Por un lado, en el ejemplo (21) falta la "s" del plural, mientras que en el (22) aparece la forma en singular en lugar de la forma plural correcta. A nivel sintáctico, la mayoría de los errores están relacionados con problemas de concordancia de número y de género. Así pues, aunque dicha concordancia existe en francés, no parece que esto ayude al alumnado a evitar errores y parece que se basa más en su conocimiento oral de la L1 que en el escrito ("restaurants" con la "s" muda, "anglais" invariable en número). Por otro lado, en el ejemplo (23) falta la tilde para la forma verbal aguda, mientras que en el (24) la tilde tampoco aparece aun tratándose de una palabra esdrújula. En estos casos producidos en el nivel ortográfico, los errores también parecen estar relacionados con la producción oral. La mayoría de estos errores son de omisión de una tilde y, aunque ello supone cambios a nivel fonético (en lo que concierne a la sílaba tónica), esto no resulta evidente para el alumnado, que parece adaptar la acentuación propia de su L1 (segunda y tercera persona del singular del futuro de indicativo en francés) o situar simplemente la sílaba tónica como si la palabra fuera francesa (tendencia a palabras Ilanas). 
Los siguientes (25 y 26) son ejemplos de hipergeneralización por falsa analogía en combinación con interferencia de la L1. Cabe mencionar que la mayoría de dichos errores se producen a nivel sintáctico por el uso erróneo de preposiciones:

(25) Podríem caminar a la muntanya i anar a [amb] vaixell pel riu.

(26) Podríem caminar a la muntanya i anar amb vaixell sobre el [pel] riu.

Los ejemplos (27) y (28) son casos de aplicación incorrecta de las normas en combinación con influencia de otra lengua aprendida (en este caso, el español):

(27) Fem experiencies [experiències] amb ratolins.

(28) La gent és molt simpàtica, el [l'] horari a la feina és ideal per a mi...

En el ejemplo (27) falta la tilde para la palabra esdrújula en catalán (la palabra es llana en español), mientras que en el (28) no se ha aplicado la regla del apóstrofo. En el corpus analizado, este tipo de errores son principalmente ortográficos con consecuencias fonéticas (27) o sintácticos (28).

\section{Conclusiones}

En este artículo se han analizado de forma tanto cuantitativa como cualitativa los errores más frecuentes en un corpus de redacciones de nivel B1 de catalán escritas por alumnado francófono. Los resultados obtenidos de los análisis permiten corroborar las tesis de Fernández (1997) y Alexopoulou (2006), dado que en el nivel B1 el alumnado parece situarse en medio de una fluctuación entre los apoyos interlingüísticos y los intralingüísticos: si bien ya comienza a utilizar conocimientos adquiridos para la misma lengua (el catalán en este caso), sigue recurriendo a su L1 y, en menor medida, a otras lenguas aprendidas, para resolver dudas surgidas al escribir en catalán. A partir de estos resultados, se pueden obtener las siguientes conclusiones:

1. Los errores que proceden de la aplicación de mecanismos intralingüísticos son los más comunes en este nivel, tanto si estos son causa única del error como en combinación con mecanismos interlingüísticos.

2. Dentro de las causas intralingüísticas, las más importantes son la aplicación incompleta de las reglas y la hipergeneralización por falsa analogía.

3. Dentro de las causas interlingüísticas, la más importante es la interferencia de la L1 seguida de la interferencia de otra lengua aprendida, especialmente si se trata de una lengua próxima a la lengua meta.

4. Las combinaciones de causas intralingüísticas e interlingüísticas más frecuentes tienden a combinar la aplicación incompleta de las reglas con la interferencia de la $L 1$, la hipergeneralización por falsa analogía también junto con la interferencia de la L1, y la aplicación incompleta de las reglas con interferencia de otra lengua aprendida.

Desde una perspectiva puramente interlingüística, el estudio permite obtener las siguientes conclusiones:

1. La interferencia de la L1 del alumnado (francés) es, por sí sola, una razón importante para la aparición de errores en catalán.

2. La interferencia de otra lengua aprendida (en este caso, el español) es también una causa importante de aparición de errores en catalán.

3. La interferencia conjunta de la L1 y otra lengua aprendida parece provocar situaciones similares a la de los errores que ocurren exclusivamente por interferencia del español.

Asimismo, desde una perspectiva puramente intralingüística se pueden obtener las siguientes conclusiones:

1. La aplicación incompleta de reglas y la hipergeneralización por falsa analogía son las causas de errores intralingüísticos más comunes. Ambas pueden darse en conjunción con causas interlingüísticas (especialmente, la interferencia de la L1) o sin ellas.

2. La aplicación incompleta de reglas suele dar lugar a errores en el nivel ortográfico con consecuencias fonéticas (uso incorrecto de tilde). 
3. La hipergeneralización por falsa analogía se da en todos los niveles del sistema.

4. La no aplicación de las restricciones de las reglas suele producirse principalmente sin que haya interferencias interlingüísticas. Se trata principalmente de errores ortográficos con consecuencias fonéticas y sintácticos.

5. La hipercorrección es un mecanismo poco común cuya incidencia es anecdótica en este nivel. Sin embargo, cuando se da, suele ocurrir en los niveles sintáctico u discursivo (uso excesivo de pronombre débiles o del modo subjuntivo).

Estas conclusiones resultan, además, interesantes y pueden arrojar luz sobre los aspectos que han de trabajarse de manera más detallada en clase a este nivel y en la preparación del examen de B1 de catalán, a saber:

1. Las reglas de acentuación y ejercicios que evidencien la distinción que existe oralmente cuando se elige una opción ortográfica y no otra.

2. Las tildes diacríticas.

3. Las reglas de concordancia de género y de número con contraejemplos que oralmente sonarían correctos en la L1 del alumnado.

4. Los apóstrofos si, siendo el alumnado francófono, ha estudiado previamente una lengua como el español, en la que no se utilizan estos signos.

5. Los verbos irregulares más frecuentes, que son regulares en francés o al menos presentan conjugaciones menos irregulares en dicha lengua.

6. Las estructuras sintácticas con preposiciones diferentes en la L1 u otra lengua aprendida.

7. Los calcos semánticos o léxicos propios de las temáticas abordadas hasta el nivel B1.

Aunque es posible que el alumnado necesite alcanzar un nivel más avanzado para constatar la integración habitual en sus redacciones de algunas de las estructuras de nivel intermedio mencionadas más arriba (Lightbown y Spada, 2018, p. 45), existen múltiples técnicas y estrategias que podrían ser útiles para combatir ya algunos de los errores recurrentes detectados. A partir de Larsen-Freeman y Anderson (2016), se proponen algunas técnicas y estrategias como ejemplo en la Tabla 9 de los Anexos. Dichas técnicas y estrategias podrían ser adaptadas según la metodología docente, las necesidades y características específicas del alumnado y las herramientas de las que dispone, los objetivos del curso, etc.

\section{Apoyos}

Querría agradecer los valiosos comentarios realizados por mis colegas Nancy Gagné (Université TÉLUQ) y Ėric Viladrich Castellanas (Université de Montréal) durante la realización de este artículo, así como la gran ayuda de Anne-Sophie Julien (SCS - Université Laval) para su componente estadístico. La consulta del corpus no hubiera sido posible sin la colaboración del Institut Ramon Llull.

\section{Referencias}

Alexopoulou, A. (2006). Los Criterios Descriptivo y Etiológico en la Clasificación de los Errores del Hablante no Nativo: una Nueva Perspectiva. Porta Linguarum, 5, 17-35. Recuperado de https://bit.ly/20mxWre.

Astolfi, J. P. (1997, 2015). L'erreur, un outil pour enseigner. Issy-les-Moulineaux. ESF éditeur.

Consejo de Europa (2001). Cadre européen commun de référence pour les langues. Didier.

Consejo de Europa (2018). Cadre européen commun de référence pour les langues. Volume complémentaire avec de nouveaux descripteurs. Recuperado de https://bit.ly/33oRL5c.

Corder, S. P. (1973). Introducing Applied Linguistics. Penguin Books.

Ellis, R. (1997). Second Language Acquisition. Oxford University Press.

Ellis, R. (2003). Task-based Language Learning and Teaching. Oxford University Press.

Ellis, R. (2008). The Study of Second Language Acquisition. Oxford University Press.

Ellis, R. \& Barkhuizen, G. (2005). Analysing Learner Language. Oxford University Press.

Erdogan, V. (2005). Contribution of Error Analysis to Foreign Language Teaching. Mersin University Journal of the Faculty of Education, 2, 261-270.

https://doi.org/10.17860/efd.22900.

Eurostat (2016). New 2016 foreign language learning statistics in the EU. Recuperado de https://bit.ly/37GYAIV.

Fernández, S. (1997). Interlengua y análisis de errores en el aprendizaje del español como lengua extranjera. Edelsa. Fernández Jódar, R. (2006). Análisis de errores léxicos, morfosintácticos y gráficos en la lengua escrita de los aprendices polacos de español (tesis doctoral). Recuperado de https://bit.ly/2Dh65|J. 
Housen, A., Kuiken, F. \& Vedder, I. (2012). Dimensions of L2 performance and proficiency: complexity, accuracy and fluency in SLA. John Benjamins.

Huensch, A., \& Tracy-Ventura, N. (2017). Understanding second language fluency behavior: The effects of individual differences in first language fluency, cross-linguistic differences, and proficiency over time. Applied Psycholinguistics, 38(4), 755-785.

https://doi.org/10.1017/s0142716416000424.

Larsen-Freeman, D. \& Anderson, M. (2016). Techniques and principles in language teaching. Oxford University Press. Lightbown, P. M. \& Spada, N. (2018). How languages are learned. Oxford University Press.

Mas, M., Vilagrasa Grandia, A. \& Bastons i Vilallonga, N. (2009). Veus 1: curs comunicatiu de català: enfocament per tasques. Publicacions de l'Abadia de Montserrat.

Skehan, P. (1998). A Cognitive Approach to Language Learning. Oxford University Press.

Verdés Prieto, G., Álvarez Verger, J., Birello, M., Mas Prats, M. \& Pérez Ventayol, M. (2018). A punt: Curs de llengua catalana (A. Vilagrasa Grandia Ed.). Publicacions de l'Abadia de Montserrat. 